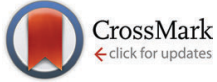

Cite this: Phys. Chem. Chem. Phys., 2014, 16, 21795

\title{
A combined IR/IR and IR/UV spectroscopy study on the proton transfer coordinate of isolated 3-hydroxychromone in the electronic ground and excited state $\dagger$
}

\begin{abstract}
A. Stamm, M. Weiler, A. Brächer, K. Schwing and M. Gerhards*
In this paper the excited state proton transfer (ESPT) of isolated 3-hydroxychromone (3-HC), the prototype of the flavonols, is investigated for the first time by combined IR/UV spectroscopy in molecular beam experiments. The IR/UV investigations are performed both for the electronically excited and electronic ground state indicating a spectral overlap of transitions of the 3-HC monomer and clusters with water in the electronic ground state, whereas in the excited state only the IR frequencies of the proton-transferred monomer structure are observed. Due to the loss of isomer and species selectivity with respect to the UV excitations IR/IR techniques are applied in order to figure out the assignment of the vibrational transitions in the $S_{0}$ state. In this context the quadruple resonance IR/UV/IR/UV technique (originally developed to distinguish different isomers in the electronically excited state) could be applied to identify the $\mathrm{OH}$ stretching vibration of the monomer in the electronic ground state. In agreement with calculations the $\mathrm{OH}$ stretching frequency differs significantly from the corresponding values of substituted hydroxychromones.
\end{abstract}

Received 9th June 2014 Accepted 13th August 2014

DOI: $10.1039 / c 4 c p 02546 f$

www.rsc.org/pccp

\section{Introduction}

The transfer of a proton after electronic excitation (excited state proton transfer, ESPT) is an intensively investigated phenomenon due to its importance in a variety of photochemical and biological processes ( $c f$. ref. 1-12). It e.g. plays an important role in the photoprotective mechanism of flavonoids, which are plant secondary metabolites. Here it serves to protect the plants against UV-B radiation. ${ }^{2,10,11}$

Within an ESPT molecule a proton-donor and a proton-acceptor group must be present. Frequently these groups already interact in the electronic ground state via e.g. hydrogen bonds. Typical arrangements are the proton transfer of a hydroxyl to a keto group, ${ }^{8,13-15}$ between a hydroxyl group and an imine group, ${ }^{16,17}$ from an amine to a keto functionality ${ }^{1}$ and between an amine and an imine functionality. ${ }^{18}$

3-HC drawn in Fig. 1 represents the basic structure of the flavonols, a subgroup of the antioxidative and photoprotective flavonoids. After electronic excitation of the normal form $(\mathrm{N})$ into a (mostly) short-lived electronically excited state $\left(\mathrm{N}^{*}\right)$ the proton migration transfers the system into the tautomeric,

TU Kaiserslautern, Fachbereich Chemie \& Research Center Optimas,

Erwin-Schroedinger-Straße 52, D-67663 Kaiserslautern, Germany.

E-mail: gerhards@chemie.uni-kl.de

$\dagger$ Electronic supplementary information (ESI) available. See DOI: 10.1039/ c4cp02546f<smiles>COc1coc2ccccc2c1=O</smiles>

cis structure b)<smiles>O=c1c([O-])coc2ccccc12</smiles>

tautomeric structure

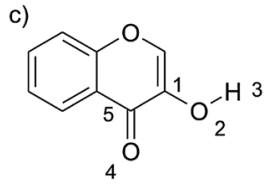

trans structure
Fig. 1 Structural isomers of 3-hydroxychromone.

electronically excited structure ( $\mathrm{T}^{*}$ ). After relaxation (e.g. via fluorescence) into the tautomeric ground state $\mathrm{T}$ the back proton transfer regenerates the originally existing $\mathrm{N}$ form in the electronic ground state so that the photocycle is closed. This typical ESPT scheme is sketched in Fig. S1 of the ESI. $\dagger$

An intensively studied representative of the class of flavonols is 3-hydroxyflavone (3-HF) which undergoes a keto-enol tautomerism after $\pi-\pi^{*}$ excitation. Investigations have been performed in solution, different matrices (e.g. ref. 19-26) and supersonic expansions. ${ }^{27-30}$

Furthermore a variety of theoretical investigations have been performed. ${ }^{31-39}$ The direct structural proof for the ESPT of isolated 3-HF in the gas phase was given by our group by applying structure sensitive combined IR/UV methods (IR/R2PI for the $S_{0}$ state and $\mathrm{UV} / \mathrm{IR} / \mathrm{UV}$ for the $\mathrm{S}_{1}$ state) under isolated molecular beam conditions. ${ }^{30}$ In comparison to the calculated vibrational frequencies of different (TD)-DFT optimised structures the presence of the tautomeric form in the electronically excited 
state could be demonstrated not only for 3-HF but also for 2-(2-naphthyl)-3-hydroxychromone (2-NHC), a derivative, differing in the naphthyl substituent on the chromone moiety. ${ }^{30}$ Beyond this, in the isolated $3-\mathrm{HF}\left(\mathrm{H}_{2} \mathrm{O}\right)_{2}$ cluster for the first time a proton wire was analysed in the electronically excited state by an IR/UV method. ${ }^{29}$

The ongoing discussion in the literature about the existence of an energy barrier for the PT (cf. e.g. ref. 21, 26, 28, 34 and 40-43), arising either from the required coplanarity of the phenyl ring with the chromone moiety ( $c f$. e.g. ref. 21 and 40) or from the $\mathrm{OH}$ bending mode modulating the proton transfer distance, ${ }^{44}$ leads us to the investigation of 3-hydroxychromone (3-HC) as the basic structure. For 3-HC the PT coordinate should be easier to describe since no further aromatic substituent exists. In former studies the ESPT for 3-HC was investigated by fluorescence spectroscopy in solution ${ }^{40,45-47}$ or in a $10 \mathrm{~K}$ argon matrix..$^{23,24,48}$ These studies mainly revealed the ESPT in 3-HC to be faster than in the case of 3-HF and the electronically excited 3-HC tautomer to be longer-lived. ${ }^{40}$

The only molecular beam investigation on 3-HC, in which the formation of water clusters was assumed to be unlikely, is a comparative fluorescence study with $3-\mathrm{HF}$ and 2 -NHC. ${ }^{28,49}$ The low frequency vibrations in the fluorescence excitation spectra of 3-HF and 2-NHC, which were absent for 3-HC, could be assigned to the torsional movement of the phenyl or naphthyl residue, respectively. Since the activation barrier for the ESPT depends on this movement the ESPT rate increases with a decrease in the size of the substituent on the $\gamma$-pyron ring which means that 3-HC shows the fastest proton transfer $\left(k_{3-\mathrm{HC}}=1.77 \times 10^{12} \mathrm{~s}^{-1}>k_{3-\mathrm{HF}}=6.55 \times\right.$ $10^{11} \mathrm{~s}^{-1}>k_{2-\mathrm{NHC}}=1.54 \times 10^{11} \mathrm{~s}^{-1}$ ).

However, new fs-UV/VIS and IR investigations in different solvents argue the converse. Here the comparison of experimental data in apolar organic and polar partially protic solvents and quantum chemical calculations suggest the involvement of a trans structure as an intermediate prior to the ESPT in the electronically excited state which is stabilised by solvent interactions. This finally effects a slower proton transfer on the picosecond time scale. Thus a substituent on the chromone unit as the phenyl group in 3-HF might even promote a faster ESPT since it hinders specific solute-solvent interactions enabling the pathway via the stabilised trans structure. ${ }^{50,51}$ Further theoretical investigations on the structure and reactivity of 3-HC are presented in ref. 32 and 52-54.

In order to achieve more information on the structure and reactivity of isolated 3-HC and defined aggregates with water we present in this paper the first molecular beam investigations of 3-HC including mass selected IR spectroscopy for the electronic ground state (IR/R2PI method) and the electronically excited state (UV/IR/UV method). Both the structurally relevant regions of the $\mathrm{OH}$ stretching and $\mathrm{CO}$ stretching/OH bending modes are analyzed. Due to the discussion of overlapping isomers/species also quadruple resonance (IR/IR) methods have to be applied, ${ }^{55,56}$ including a new variant in order to distinguish different species in the electronic ground state. The comparison with calculated vibrational frequencies of different (TD)-DFT optimised structures allows structural assignments. Those techniques/approaches represent ideal tools to investigate the intrinsic structural properties of cooled isolated molecules without the influence of an environment. These detailed investigations give comprehensive information on the hydrogen-bonding within the molecule (and its cluster with water) offering the possibility to perform direct comparisons with the results obtained for substituted 3-hydroxychromones like 3-HF and 2-NHC.

\section{Experimental set-up}

A detailed description of the experimental set-up is given by former publications, $c f$. e.g. ref. 56. Thus only the main aspects for understanding the measurements should be explained here. All spectra were recorded by using a vacuum apparatus basically consisting of a differentially pumped time-of-flight mass spectrometer and a pulsed valve (General Valve Iota One, $500 \mu \mathrm{m}$ orifice) for skimmed jet expansion. The investigated 3-HC was synthesised by our group. The two-step synthesis is described in the ESI. $\dagger$ The sample was heated for the jet expansion to $130{ }^{\circ} \mathrm{C}$ and helium and neon were used as carrier gases (1800 mbar). The measurements presented in this paper refer to helium expansion conditions. The results obtained for neon expansion support our assignment but they yield no new results regarding the interpretation. All aspects concerning the neon expansion experiments are given in the ESI. $\dagger$

To perform the quadruple resonance techniques (IR/IR/R2PI and IR/UV/IR/UV, details $c f$. ref. 56) four independent laser systems are required. The two UV wavelengths for excitation and ionisation were generated by two frequency-doubled dye lasers (Sirah Cobra Stretch and Sirah Precision Scan); the FWHM of the UV system is about 6 ns. The two independent IR laser systems ${ }^{57}$ have the same basic set-up. In a $\mathrm{LiNbO}_{3}$ crystal the IR light in the region of $2800-3800 \mathrm{~cm}^{-1}(3.57-2.63 \mu \mathrm{m})$ is generated by a first difference frequency mixing (DFM1) of the fundamental of a seeded Nd:YAG laser and the output of a dye laser (Sirah Precision Scan) pumped by the second harmonic of the same Nd:YAG laser. This IR radiation is further amplified in a second $\mathrm{LiNbO}_{3}$ crystal by an optical parametric amplification (OPA) of the output of the DFM1 process and the fundamental of the Nd:YAG laser. The second IR laser system offers the possibility to yield a further amplification of the IR output by introducing a second amplifier stage. Both IR laser systems have a pulse width (FWHM) of 8-10 ns. The wavelength regions of CO stretching and $\mathrm{OH}$ bending modes are covered by a third non-linear process (DFM II, available in the region from about 4.5 to $16 \mu \mathrm{m}$ ). Here the signal (5398-5564 $\left.\mathrm{cm}^{-1}\right)$ and idler $\left(4009-3834 \mathrm{~cm}^{-1}\right)$ of the OPA process are mixed in an $\mathrm{AgGaSe}_{2}$ crystal generating radiation from 1380 to $1730 \mathrm{~cm}^{-1}$.

The time delay of the two UV lasers was optimised to $6.8 \mathrm{~ns}$ since this yielded the maximum ion signal, the best two-colour effect and a sufficient delay to insert the IR laser for all measurements in the electronically excited state. To measure the IR spectra of the electronic ground state the IR laser was fired $50 \mathrm{~ns}$ prior to the UV excitation laser. For the UV/IR/UV spectra the IR laser was fired $4 \mathrm{~ns}$ after the UV excitation laser. In the 
case of the IR/IR/R2PI measurements the first fixed IR burn laser was fired 100 ns before the UV excitation. The second, scanned IR laser followed the IR burn laser after 50 ns. For the IR/UV/IR/UV spectra the delay of the fixed IR burn laser was set to a delay time of $50 \mathrm{~ns}$ prior to the UV excitation laser whereas the scanned IR laser was delayed to fire $4 \mathrm{~ns}$ after the UV excitation laser.

\section{Theory}

The structures of the 3-hydroxychromone monomer ( $c f$. Fig. 1) in the electronic ground state and the first electronically excited state were determined by geometry optimisation at the DFT and TD-DFT levels of theory using Gaussian09. ${ }^{58}$ The B3LYP functional as implemented in Gaussian09 and the TZVP basis set were chosen. The SCF convergence and geometry convergence thresholds were set to the "tight" criterion. All energies were corrected for harmonic vibrational zero-point energies. The calculated vibrational frequencies for the structures presented here were real, indicating minima on the potential energy surface. To take anharmonic effects into account the calculated vibrational frequencies were scaled with the factor of 0.99 for the CO region and 0.9613 for the $\mathrm{OH}$ stretching region. ${ }^{29,30}$

The calculated cis structure in the electronic ground state $\left(\mathrm{S}_{0}\right)$ with a stabilising intramolecular hydrogen bond between the carbonyl and hydroxyl functionalities is the most stable isomer (cf. Fig. 1a). The corresponding trans structure $\left(\mathrm{S}_{0, t}, c f\right.$. Fig. 1c) and the proton-transferred tautomer form $\left(\mathrm{S}_{0}{ }^{\prime}, c f\right.$. Fig. 1b) are $2915 \mathrm{~cm}^{-1}\left(35 \mathrm{~kJ} \mathrm{~mol}^{-1}\right)$ and $5489 \mathrm{~cm}^{-1}\left(66 \mathrm{~kJ} \mathrm{~mol}^{-1}\right)$ less stable. In the electronically excited state, the relationship changes. Here the proton-transferred tautomeric structure $\left(S_{1}{ }^{\prime}\right)$ represents the minimum structure whereas the non-tautomeric cis $\left(\mathrm{S}_{1}\right)$ and trans structures $\left(\mathrm{S}_{1, t}\right)$ are, respectively, $4050 \mathrm{~cm}^{-1}\left(49 \mathrm{~kJ} \mathrm{~mol}^{-1}\right)$ and $4339 \mathrm{~cm}^{-1}\left(52 \mathrm{~kJ} \mathrm{~mol}^{-1}\right)$ higher in relative energy.

The most important geometry parameters for the different 3-HC structures for both electronic states are listed in Table 1. The most important aspects regarding the calculated geometries are: (a) all structures are planar in the electronic ground and excited state and (b) the proton transfer can be followed by changing the $r_{\mathrm{O} 2-\mathrm{H} 3}$ and $r_{\mathrm{H} 3-\mathrm{O} 4}$ distances. The vertical electronic excitation of the cis structure $\mathrm{S}_{0}$ to the electronically excited state $\mathrm{S}_{1}$ effects an elongation of $r_{\mathrm{O} 2-\mathrm{H} 3}$ and a shortening of $r_{\mathrm{H} 3-\mathrm{O} 4}$

Table 1 Geometry parameters obtained for the 3 different structures of $3-\mathrm{HC}$ in the $\mathrm{S}_{0}$ and $\mathrm{S}_{1}$ state calculated at the DFT and TD-DFT levels of theory (B3LYP/TZVP). The bond lengths are given in $\AA$ and the bond angles in degrees

\begin{tabular}{|c|c|c|c|c|c|c|}
\hline & \multicolumn{2}{|c|}{ cis structure } & \multicolumn{2}{|c|}{ Tautomeric structure } & \multicolumn{2}{|c|}{ trans structure } \\
\hline & $\mathrm{S}_{0}$ & $\mathrm{~S}_{1}$ & $\mathrm{~S}_{0}{ }^{\prime}$ & $\mathrm{S}_{1}^{\prime}$ & $\mathrm{S}_{0, t}$ & $\mathrm{~S}_{1, t}$ \\
\hline$r_{\mathrm{C} 1-\mathrm{O} 2}$ & 1.351 & 1.319 & 1.265 & 1.335 & 1.359 & 1.370 \\
\hline$r_{\mathrm{O} 2-\mathrm{H} 3}$ & 0.976 & 1.002 & 1.812 & 2.143 & 0.965 & 0.964 \\
\hline$r_{\mathrm{H} 3-\mathrm{O} 4}$ & 2.097 & 1.871 & 1.008 & 0.975 & 3.684 & 3.649 \\
\hline$r_{\mathrm{O} 4-\mathrm{C} 5}$ & 1.232 & 1.248 & 1.312 & 1.341 & 1.220 & 1.313 \\
\hline$r_{\mathrm{C} 5-\mathrm{C} 1}$ & 1.461 & 1.510 & 1.449 & 1.414 & 1.473 & 1.408 \\
\hline$\Phi_{\mathrm{C} 1-\mathrm{O} 2-\mathrm{H} 3}$ & 105.1 & 102.2 & 84.2 & 81.5 & 110.5 & 110.3 \\
\hline$\Phi_{\mathrm{O} 2-\mathrm{H} 3-\mathrm{O} 4}$ & 116.4 & 123.4 & 125.2 & 114.7 & 6.8 & 6.0 \\
\hline$\Phi_{\mathrm{H} 3-\mathrm{O} 4-\mathrm{C} 5}$ & 82.4 & 86.0 & 101.1 & 106.3 & 58.9 & 59.1 \\
\hline
\end{tabular}
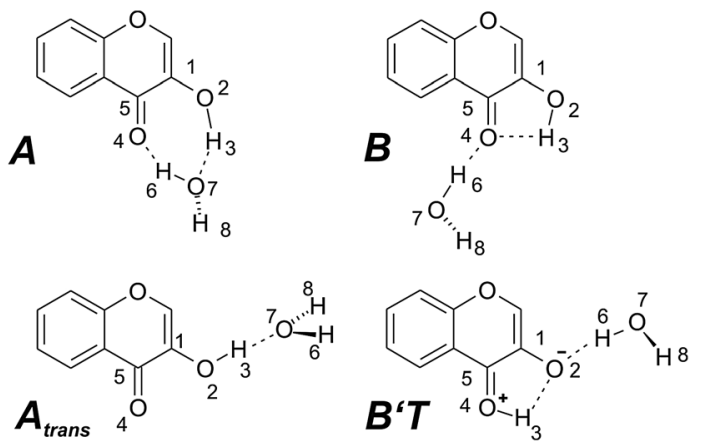

Fig. 2 Possible structures of 3-hydroxychromone with one water molecule including the two most stable arrangements $A$ and $B$. For further structures cf. ESI. $\dagger$

distances, which finally ends up in the proton-transferred (tautomeric) form $\mathrm{S}_{1}{ }^{\prime}$. It is just the other way around for going from the $S_{1}{ }^{\prime}$ to the $S_{0}{ }^{\prime}$ state structure. In the back proton transfer the hydrogen bond between $\mathrm{O} 2-\mathrm{H} 3$ is reconverted to a covalent bond whereas for $\mathrm{H} 3-\mathrm{O} 4$ it is elongated to a hydrogen bond.

Since the IR/R2PI spectrum of the 3-HC monomer showed vibrations which were suspected to arise from a UV generated fragmentation of hydrates (details: $c f$. Results and discussion) the possible structures for clusters with one and two water molecules were calculated for the electronic ground state. For the most stable monohydrate the water molecule is inserted between the carbonyl and hydroxyl groups of the chromone unit (A in Fig. 2). In the second-most stable isomer, which is about $840 \mathrm{~cm}^{-1}\left(10 \mathrm{~kJ} \mathrm{~mol}^{-1}\right)$ less stable, the water molecule is merely hydrogen-bonded to the $\mathrm{CO}$ group (B in Fig. 2). In the case of the $\mathrm{A}_{\text {trans }}$ structure $\left(3107 \mathrm{~cm}^{-1}, 37 \mathrm{~kJ} \mathrm{~mol}^{-1}\right.$ less stable, $c f$. Fig. 2) water is hydrogenbonded to the trans structure of the monomer. Additionally, further arrangements with water attached to the trans structure and the tautomeric form of the monomer were calculated ( $c f$. Fig. S2 in the ESI $\dagger$ ). Among these structures the tautomeric $\mathrm{B}^{\prime} \mathrm{T}$ arrangement $\left(5484 \mathrm{~cm}^{-1}, 66 \mathrm{~kJ} \mathrm{~mol}^{-1}\right.$ less stable) will be mentioned later; it is shown in Fig. 2. Furthermore structures with water being attached to the oxygen atom of the chromone unit like structure $\mathrm{C}\left(2070 \mathrm{~cm}^{-1}, 25 \mathrm{~kJ} \mathrm{~mol}^{-1}\right.$ less stable) and to the oxygen atom of the hydroxyl group like structure $\mathrm{B}^{\prime},\left(1312 \mathrm{~cm}^{-1}\right.$, $16 \mathrm{~kJ} \mathrm{~mol}^{-1}$ less stable) are illustrated in Fig. S2 (ESI $\dagger$ ).

The most important geometry parameters for the monohydrate isomers derived from calculations for both the electronic ground and excited state are listed in Tables S1 and S2, ESI. $\dagger$ Additionally structures of clusters with two water molecules and the corresponding most important geometry parameters are given in Fig. S5 and Table S3 of the ESI. $\dagger$

\section{Results and discussion}

A mass selective two-colour R2PI spectrum of 3-HC has been recorded in the range of $30210-31600 \mathrm{~cm}^{-1}$ ( $c f$. Fig. 3). A one-colour R2PI spectrum could not be obtained since in this region of possible excitations the energy of two UV photons of the excitation laser does not exceed the ionisation potential. 


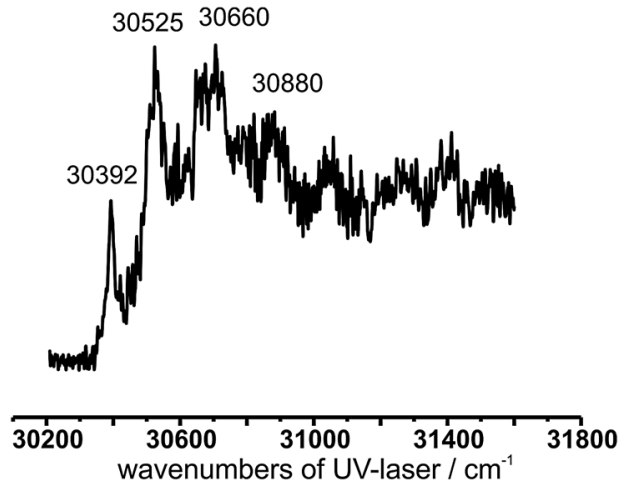

Fig. 3 R2PI spectrum of 3-hydroxychromone.

The R2PI spectrum is characterised by a broad unstructured background and an electronic origin at $30392 \mathrm{~cm}^{-1}$ which is in agreement with the origin of the laser induced fluorescence (LIF) spectrum of ref. 28. In order to get an assignment on the structure and proton transfer (PT) coordinate of 3-HC in different electronic states the R2PI spectrum is taken as a basis for further double, triple and quadruple resonance experiments (see below). An estimation of the ionization potential can be obtained by recording an ion current spectrum, for which the UV excitation laser is fixed and the ionizing laser is scanned. The recorded ion current spectrum shows no sharp increase indicating that the measured vertical ionization potential (IP) cannot directly be compared to the calculated adiabatic one.

From the ion current spectrum an IP can be estimated that is lower than $71350 \mathrm{~cm}^{-1}$, i.e. the measured vertical and the calculated adiabatic IPs differ by about $5100 \mathrm{~cm}^{-1}$. It should be mentioned that these deviations are very similar to the one obtained for 3-HF ( $c f$. ref. 30: difference between experimental and theoretical IPs is about $5700 \mathrm{~cm}^{-1}$ ). In contrast to the results obtained for 3-HF ( $c f$. ref. 29 and 30) no ion signals of clusters with water could be observed if helium is used as a carrier gas. This result was independent of the chosen excitation and ionisation wavelengths. The combined IR/UV investigations given in the next sections will show if no clusters are formed or if they fragment after electronic excitation.

The IR/R2PI spectra in the spectral region of both the $\mathrm{OH}$ stretching vibrations as well as the carbonyl stretching and $\mathrm{OH}$ bending modes obtained via the mass trace of 3-HC are depicted in Fig. 4; the spectra are identical for the different possible UV excitation wavelengths indicated in the R2PI spectrum. In the $\mathrm{OH}$ stretching region four transitions at 3268, 3410, 3461 and $3715 \mathrm{~cm}^{-1}$ are observed. The small shoulder at about $3300 \mathrm{~cm}^{-1}$ of the transition at $3268 \mathrm{~cm}^{-1}$ may be attributed to an overtone of the strong experimental transition at $1651 \mathrm{~cm}^{-1}$. However, in the spectral region of the $\mathrm{OH}$ stretching modes only one transition of the hydroxyl group of 3-HC is expected. Especially the transition at $3715 \mathrm{~cm}^{-1}$, which typically belongs to a free $\mathrm{OH}$ stretching mode of a water molecule with one non-hydrogenbonded $\mathrm{OH}$ group, is a clear indication that not only pure 3-HC but also cluster(s) of water are indirectly investigated - although no mass signals of hydrates could be observed. The fact that IR bands of hydrates can be recorded in the IR/R2PI spectrum via the monomer ion signal is due to a UV generated fragmentation of the cluster(s) (detailed explanations for this frequently occurring behavior are given in the ESI $\dagger$ ). These investigations already have shown the important conclusion that in contrast to previous assumptions derived from pure fluorescence spectra (cf. ref. 28 and 49) clusters with water are formed.

In order to explain the presence of clusters with water in the IR spectra of 3-HC and to get a further assignment, the IR spectra are first compared with the values calculated for the different possible structures of pure 3-HC (cf. Fig. 1). In Fig. 4 the calculated IR spectra based on the harmonic approach are shown. Obviously the most stable cis structure (Fig. 1a) fits best to the experimental spectrum both in the region of $\mathrm{OH}$ bending

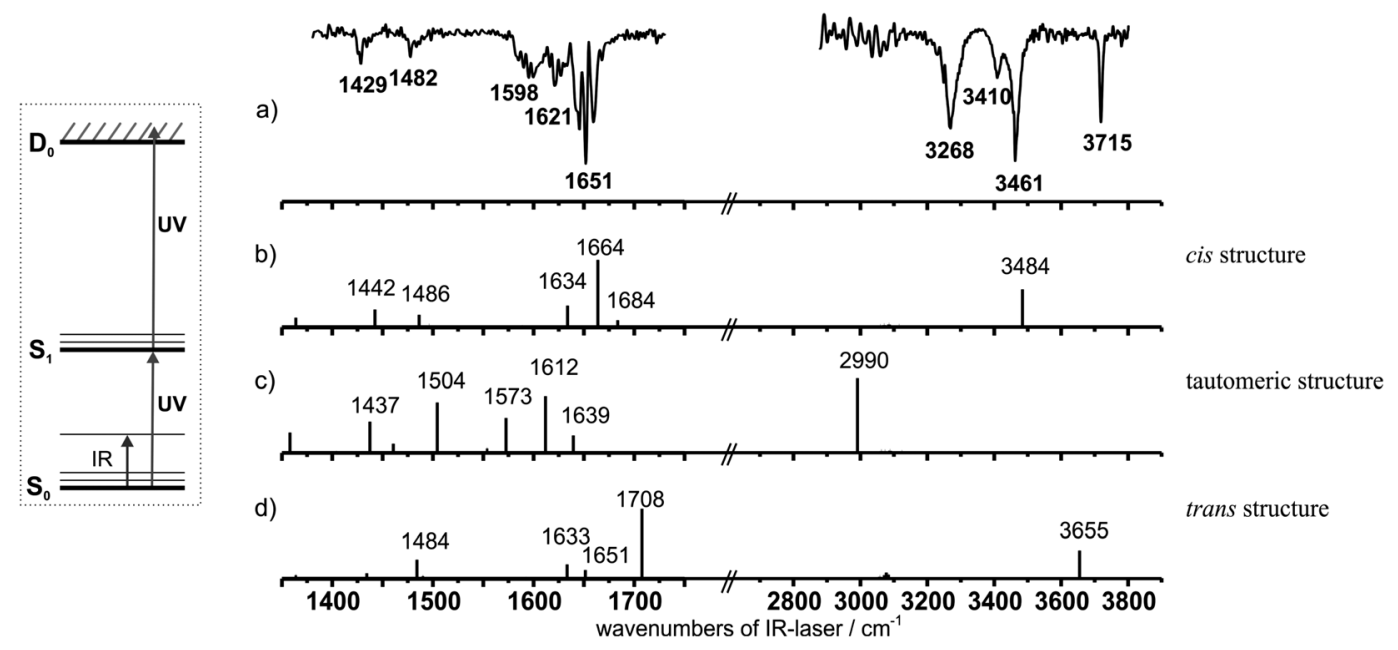

Fig. 4 (a) Experimental IR/R2PI spectrum of 3-hydroxychromone in the electronic ground state $\mathrm{S}_{0}$ with the excitation laser at $30525 \mathrm{~cm}^{-1}$ and the ionisation laser at $45351 \mathrm{~cm}^{-1}$. (b) Calculated IR spectra (B3LYP/TZVP, scaling factor OH stretching region: 0.9613 ; scaling factor $\mathrm{CO}$ stretching/OH bending region: 0.99 ) of the $S_{0}$ state cis structure, (c) of the tautomeric structure and (d) of the trans structure. (For a better illustration the calculated intensities in the $\mathrm{OH}$ stretching region were doubled.) 
Table 2 Calculated $\mathrm{OH}$ stretching vibrations for the different structural isomers of 3-HC in the electronic ground state (DFT/B3LYP/TZVP) and the electronically excited state (TD-DFT/B3LYP/TZVP). Scaling factor: 0.9613

Experiment cis structure Tautomeric structure trans structure

\begin{tabular}{lllllllll} 
& $\mathrm{S}_{0}$ & $\mathrm{~S}_{1}$ & $\mathrm{~S}_{0}$ & $\mathrm{~S}_{1}$ & $\mathrm{~S}_{0}{ }^{\prime}$ & $\mathrm{S}_{1}{ }^{\prime}$ & $\mathrm{S}_{0, t}$ & $\mathrm{~S}_{1, t}$ \\
\hline 3-HC & 3461 & 3401 & 3484 & 3080 & 2990 & 3514 & 3655 & 3670 \\
3-HF & 3276 & 3350 & 3403 & 2928 & 3058 & 3436 & 3622 & 3624 \\
2-NHC & 3280 & 3362 & 3398 & 3035 & 3091 & 3421 & 3611 &
\end{tabular}

and carbonyl stretching modes as well as in the $\mathrm{OH}$ stretching region. The $\mathrm{OH}$ stretching modes calculated for the trans and tautomeric structures are either too low or too high in energy with respect to the experimentally observed possible $\mathrm{OH}$ stretching transitions between 3268 and $3461 \mathrm{~cm}^{-1}$ (cf. Table 2).

According to the harmonic calculations the transition at $3461 \mathrm{~cm}^{-1}$ seems to fit best but it is known from 3-HF that anharmonic effects can lead to a significant reduction of this value. In the case of 3-HF the experimentally observed $\mathrm{OH}$ stretching frequency is $3276 \mathrm{~cm}^{-1}$ which is closer to the transition at $3268 \mathrm{~cm}^{-1}$ of 3-HC. In order to yield an assignment, different experimental strategies have been chosen. In one attempt IR/R2PI spectra were recorded in a way that the IR laser was fixed on the different transitions of the IR spectrum and the UV laser is scanned in the region of the R2PI spectrum ( $c f$. Fig. 5). If the IR photon excites 3-HC other R2PI transitions should be influenced (depleted) than for the case that hydrates are vibrationally excited. The spectra obtained via the transitions at 3268,3410 and $3461 \mathrm{~cm}^{-1}$ show that all UV transitions of the original R2PI spectrum are affected in the same way, i.e. as far as pure 3-HC and clusters with water are observed all species have very similar UV spectra so that the UV excitation is not isomer and species selective in this case.

If no isomer or species selectivity is given by the UV excitation, the application of an IR/IR/R2PI experiment ${ }^{55,56}$ may retain the isomer/species selectivity. In this case the excitation of one isomer/species with a frequency-fixed IR burn laser has an effect on all IR transitions that belong to the same isomer/species in the subsequently measured IR/R2PI spectrum. It should be mentioned that in this application the IR/IR spectra of clusters are measured on the monomer mass trace. This complication can avoid any quantitative analysis, i.e. only a qualitative correlation between peaks that belong together can be given.
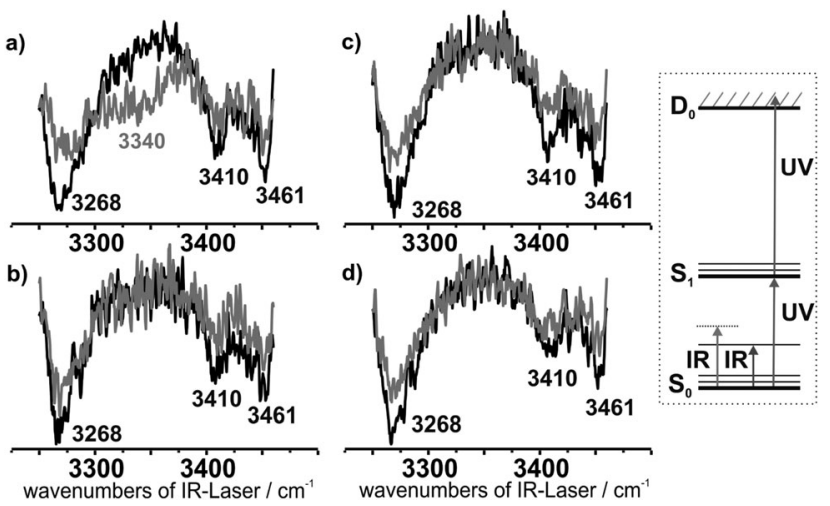

Fig. 6 IR/IR/R2PI spectra of 3-hydroxychromone by using different burn laser frequencies at (a) $3268 \mathrm{~cm}^{-1}$, (b) $3410 \mathrm{~cm}^{-1}$, (c) $3461 \mathrm{~cm}^{-1}$ and (d) $3715 \mathrm{~cm}^{-1}$ for the first IR photon. The IR/IR/R2PI spectra are given in grey and the corresponding IR/R2PI spectra (without using the burn laser) are given in black.

For the 3-HC investigations the burn laser was set to one of the four transitions at $3268,3410,3461$ or $3715 \mathrm{~cm}^{-1}$ ( $c f$. Fig. 6). Unfortunately, the spectra show (like in the spectra of Fig. 5) that all transitions influence one other. Especially the excitation of the "water" characteristic band at $3715 \mathrm{~cm}^{-1}$ seems to correlate with all other transitions, i.e. all vibrations should be associated with clusters. On the other hand it cannot be excluded that one of the transitions is an overlap of a vibration belonging to pure 3-HC and a cluster with water. The IR/IR method can only be species selective if at least one IR transition exclusively belongs to the monomer. Thus, on the basis of the IR/IR/R2PI experiments a final assignment for the vibration in the $\mathrm{S}_{0}$ state cannot be given, but further information can be obtained from the investigation of the electronically excited state and on the basis of these results we can draw further conclusions for the interpretation of the electronic ground state IR spectra.

The electronically excited state is analysed by applying the $\mathrm{UV} / \mathrm{IR} / \mathrm{UV}$ method both in the region of the $\mathrm{OH}$ stretching modes and the region of the carbonyl stretching and $\mathrm{OH}$ bending vibrations (cf. Fig. 7). In the region of the $\mathrm{OH}$ stretching vibrations only one transition at $3401 \mathrm{~cm}^{-1}$ is observed which could be a hint that only the monomer species (with one $\mathrm{OH}$ stretching frequency) is measured in the electronically excited state. (e.g. in the case of the monohydrated cluster three transitions in the
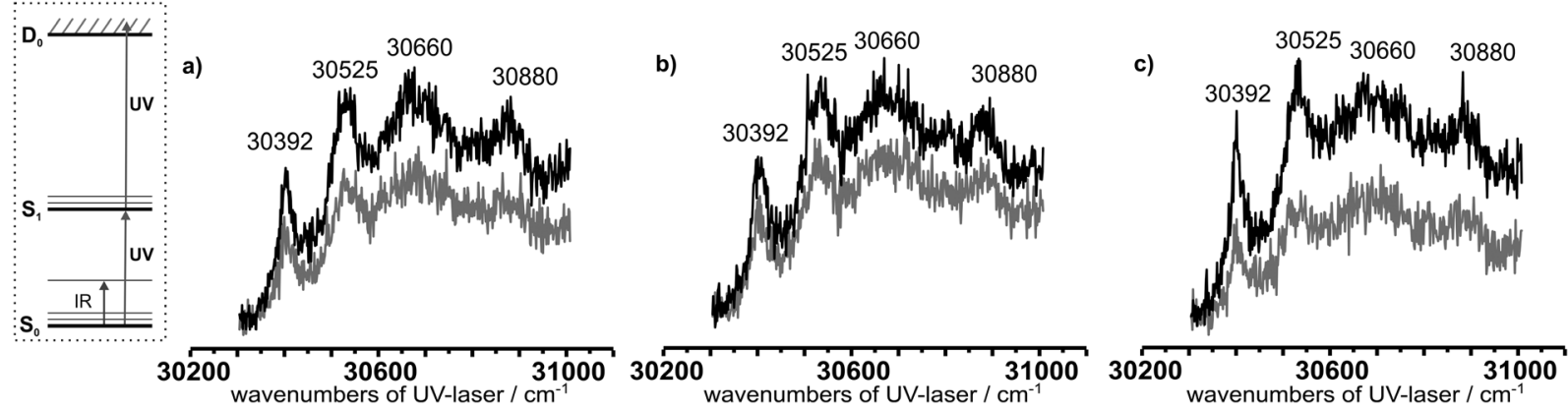

Fig. 5 IR/R2PI spectra of 3-hydroxychromone by using different burn laser frequencies at (a) $3268 \mathrm{~cm}^{-1}$, (b) $3410 \mathrm{~cm}^{-1}$ and (c) $3461 \mathrm{~cm}^{-1}$ for the first IR photon. The IR/R2PI spectra are given in grey and the corresponding R2PI spectra are given in black. 


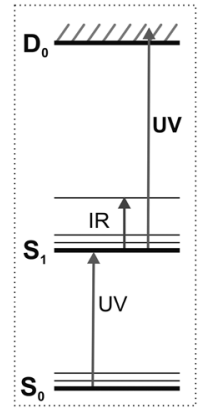

a)
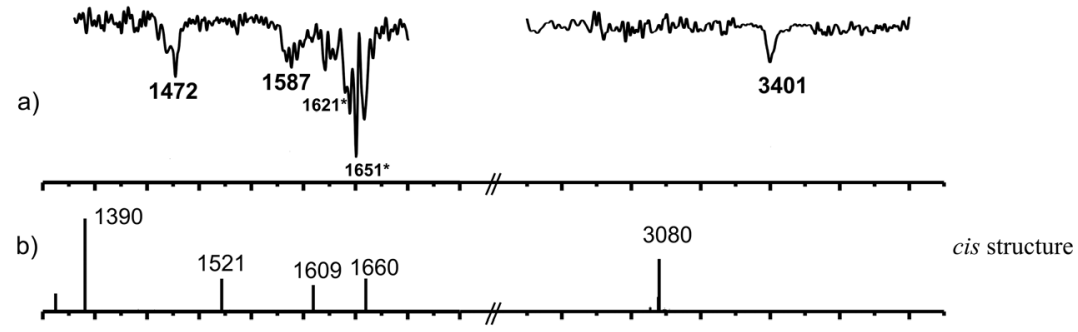

c)

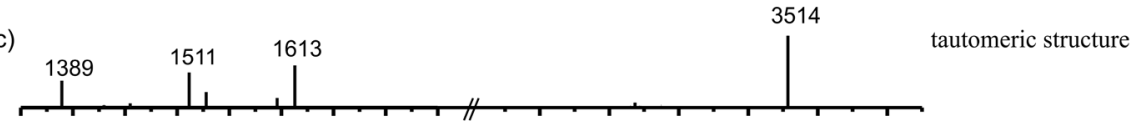

d)

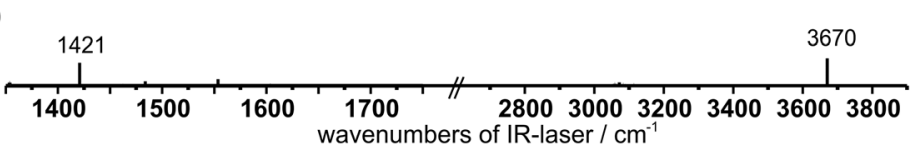

trans structure

Fig. 7 (a) Experimental spectrum of 3-hydroxychromone in the $S_{1}$ state. The asterisks indicate vibrations from the $S_{0}$ state due to a partial temporal overlap of excitation (UV) and IR laser. (b)-(d) Calculated stick spectra of the $S_{1}$ state (TD-DFT/B3LYP/TZVP; for scaling factors cf. Fig. 4): (b) cis structure, (c) tautomeric structure, and (d) trans structure. (For a better illustration the calculated intensities in the $\mathrm{OH}$ stretching region were doubled.)

range from 2700 to $3800 \mathrm{~cm}^{-1}$ should be observed, $c f$. calculated spectra in Fig. S4, ESI.†) By comparing the value of $3401 \mathrm{~cm}^{-1}$ with the experimentally obtained frequencies for the tautomeric forms of 3-HF $\left(3350 \mathrm{~cm}^{-1}\right)$ and 2-NHC $\left(3362 \mathrm{~cm}^{-1}\right)^{30}$ as well as their corresponding calculated values ( $c f$. Table 2$)$, a similar deviation between calculated and experimentally determined frequencies is observed resulting from the anharmonicity of the $\mathrm{OH}$ stretching vibration. The $\mathrm{OH}$ stretching vibrations calculated for the less stable cis and trans forms (cf. Section III) are either too low or too high compared to the experimentally observed value ( $c f$. Table 2 and Fig. 7). This interpretation is further supported by the spectra obtained in the CO stretching/ $\mathrm{OH}$ bending region. The two vibrational transitions observed at 1472 and $1587 \mathrm{~cm}^{-1}$ exhibit a similar distance as the two calculated frequencies at 1511 and $1613 \mathrm{~cm}^{-1}$. Compared to the $\mathrm{OH}$ stretching modes the absolute values of the calculated frequencies are higher due to their anharmonicities. According to the calculations performed in this spectral region for the cis and trans arrangements, both structures do not fit with respect to their peak positions and expected intensities ( $c f$. Fig. 7). Thus an unambiguous assignment of the tautomeric form in the excited state $\left(\mathrm{S}_{1}{ }^{\prime}\right)$ is obtained so that similar to 3-HF and 2-NHC an excited state proton transfer could also be proven for isolated 3-HC.

As mentioned before, the most striking observation is that (in contrast to the electronic ground state) no vibrations of clusters with water are observed in the IR spectrum of the electronically excited state via the 3-HC mass trace. This is a clear indication that the clusters with water fragment after UV excitation. If the hydrates fragmented during the ionisation process, additional hydrate bands should also be visible in the $\mathrm{UV} / \mathrm{IR} / \mathrm{UV}$ spectrum via the 3-HC mass trace. Since a complete fragmentation of the cluster may be less probable it can furthermore be assumed that the electronically excited state of the clusters does not live long enough for ionisation with a ns laser after electronic excitation (cf. e.g. ref. 59 and 60). It could be assumed that the broad background of the R2PI spectrum (cf. Fig. 3) with a width of several hundreds of wavenumbers may be correlated to the clusters with short lifetimes in the fs regime. Due to (a) the form of the R2PI spectrum (increasing signal), (b) the fragmentation of the hydrates and (c) the resulting overlap of 3-HC and hydrate peaks a fitting of individual peaks with Lorentzian line shapes (like in ref. 61) is not unambiguous. Nevertheless, the width of possible peaks between 20 and a few hundred wavenumbers indicate the presence of short-lived species.

As a further consequence of a short excited state lifetime the monomer and the hydrates cannot be discriminated by their ionisation potential as in the case of $3-\mathrm{HF}^{29,30}$ This was decisively useful to determine the 3-HF monomer band and discriminate it from the spectra of the cluster species which can also be observed on the mass traces of the clusters.

From the UV/IR/UV spectrum of 3-HC the formation of the tautomeric structure in the electronically excited state $\left(\mathrm{S}_{1}{ }^{\prime}\right)$ can be derived but according to our investigations with respect to the $S_{0}$ state the question remains if (a) no monomer is observed in the $\mathrm{S}_{0}$ state, i.e. all transitions arise from clusters with water which fragment in the $S_{1}$ state or if (b) a monomer transition overlaps with one of the IR transitions arising from a hydrate. In the case of the dihydrated 3-HF cluster we introduced a new quadruple resonance technique (the IR/UV/IR/UV method ${ }^{56}$ ) in order to distinguish two different isomers within the electronically excited state if the UV excitation is not isomer selective. In the inset of Fig. 8 the method is shown schematically: The first IR laser depletes one isomer in the electronic ground state, thus (almost) only the second isomer is observed in the IR spectrum of the electronically excited state.

In the case of $3-\mathrm{HC}$ the IR/UV/IR/UV technique could be ideally suited to distinguish different species like the monomer and hydrates in the electronic ground state which have the same UV excitation energy and lead to the same species (monomer of 3-HC) in the excited state. This could work in the following way: the frequency-fixed IR burn laser (first IR laser) is set to a vibrational transition of the $\mathrm{S}_{0}$ state and a UV/IR/UV spectrum 
a)

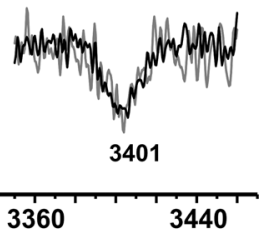

c)

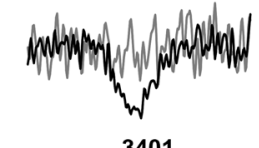

3360
wavenumbers of IR-laser $/ \mathrm{cm}^{-1}$

b)
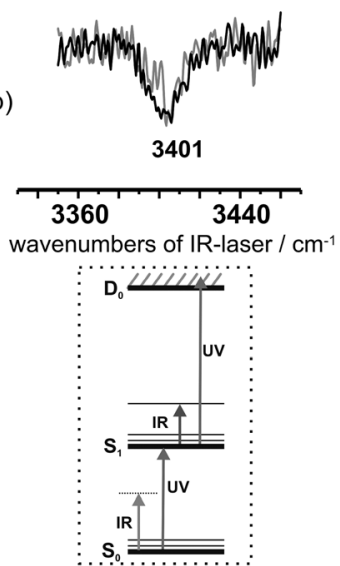

Fig. $8 \mathrm{IR} / \mathrm{UV} / \mathrm{RR} / \mathrm{UV}$ spectra of 3-hydroxychromone by using different burn laser frequencies at (a) $3268 \mathrm{~cm}^{-1}$, (b) $3410 \mathrm{~cm}^{-1}$ and (c) $3461 \mathrm{~cm}^{-1}$ for the first IR photon. The IR/UV/IR/UV spectra are given in grey and the corresponding UV/IR/UV spectra are given in black.

is recorded. If the IR burn laser exclusively excites a cluster with water in the electronic ground state, the monomer band(s) in the IR spectrum of the $S_{1}$ state should still be conserved. If the IR burn laser excites both the cluster with water and the monomer in the electronic ground state, then (almost) no IR signal should be observed in the electronically excited state. In this case the relevant monomer species is depleted in the electronic ground state. In Fig. 8, the IR/UV/IR/UV spectra (normalised to the UV/IR/UV spectra) after excitation of the transitions at 3268,3410 and $3461 \mathrm{~cm}^{-1}$ are shown. These spectra indicate clearly that the electronic ground state vibration at $3461 \mathrm{~cm}^{-1}$ must result from an overlap of more than one species, since - in contrast to the other IR/UV/IR/UV spectra the $S_{1}$ state vibration completely vanishes. Furthermore one of these overlapping species only has one $\mathrm{OH}$ transition in the spectrum of the electronic ground state at $3461 \mathrm{~cm}^{-1}$. Since this can only be the case for the monomer species the electronic ground state band at $3461 \mathrm{~cm}^{-1}$ can clearly be identified as the vibration of the monomer. By comparing this assignment with the one obtained for 3-HF $\left(3276 \mathrm{~cm}^{-1}\right)$ and 2 -NHC $\left(3280 \mathrm{~cm}^{-1}\right)$ in the $S_{0}$ state the vibrational frequency seems to be surprisingly high. On the other hand it has to be taken into account that in the case of $3-\mathrm{HC}$ already the calculated harmonic frequency is about $100 \mathrm{~cm}^{-1}$ higher compared to $3-\mathrm{HF}$ and 2-NHC. Finally one-dimensional vibrational calculations for 3-HC performed on the potential obtained from a step-wise elongation along the $\mathrm{OH}$ stretching normal mode yield an excellent agreement between calculated $\left(3450 \mathrm{~cm}^{-1}\right)$ and experimentally observed value of $3461 \mathrm{~cm}^{-1}$. The detailed theoretical analysis to describe the proton transfer coordinate performed on $1 D, 2 D$ and $3 D$ potentials is given in another publication. ${ }^{62}$

After assigning the transition at $3461 \mathrm{~cm}^{-1}$ to the $\mathrm{OH}$ stretching mode of the monomer, the transitions at $3715,3410,3268 \mathrm{~cm}^{-1}$ and again the overlapping mode at $3461 \mathrm{~cm}^{-1}$ still belong to clusters with water. Thus we compared the spectroscopically obtained results with calculations performed for clusters with one water molecule ( $c f$. Fig. 9). The comparison of the calculated

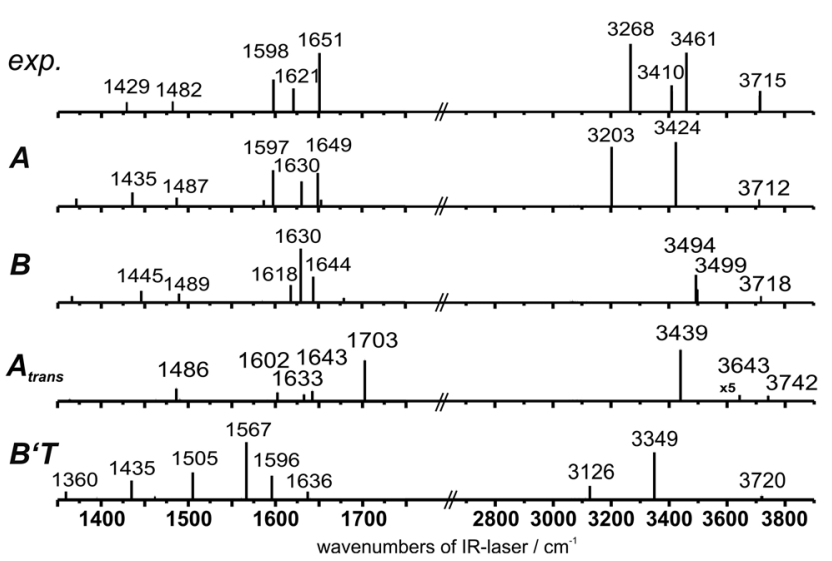

Fig. 9 The upper trace schematically depicts the experimental $S_{0}$ state spectrum (cf. Fig. 4) via the ion signal of 3-hydroxychromone as stick spectrum, which is compared to the calculated stick spectra of different monohydrate isomers (B3LYP/TZVP, for scaling factors, see Fig. 4.) For a better illustration the calculated intensities in the $\mathrm{OH}$ stretching region were doubled.

stick spectra of $3-\mathrm{HC}\left(\mathrm{H}_{2} \mathrm{O}\right)_{1}$ with the experimentally observed IR transitions on the monomer mass show a very good agreement with transitions calculated for the two most stable forms of the monohydrated cluster (A and B). The general assignment is in agreement with the discussion on the monohydrated cluster of 3-HF for which also an overlap of the two most stable arrangements is discussed. ${ }^{29}$ Therefore all transitions in the IR/R2PI spectrum of 3-HC could be assigned, resulting from the monomer as well as from fragmenting monohydrated clusters. (For further description of the vibrations, $c f$. Table S4, ESI $\dagger$. In the case of $\mathrm{Ne}$ expansion larger clusters can be formed but these do not lead to the vibrational transitions observed on the monomer trace. For further information, $c f$. ESI $\dagger$ Fig. S7, and comments).

Finally a further interesting feature should be addressed which can be found in the IR/IR/R2PI spectrum with a burn laser at $3268 \mathrm{~cm}^{-1}$ (cf. Fig. 6a). An additional band occurs at around $3340 \mathrm{~cm}^{-1}$ which was not recorded in the original IR/R2PI spectrum. The transition at $3268 \mathrm{~cm}^{-1}$ in the IR/R2PI spectrum is assigned to the monohydrated cluster A ( $c f$. Fig. 9). One possibility could be that an excitation at $3268 \mathrm{~cm}^{-1}$ may (at least partially) lead to a structural rearrangement of the cluster. With a second IR photon which follows the first one in an IR/IR/R2PI spectrum the new structure would be analysed by a further decrease of the ion signal if an IR transition of this structure is excited. IR-induced structural reorganisations are known from population transfer spectroscopy introduced by Zwier and coworkers (e.g. ref. 63). In their chosen examples the population is redistributed between different isomers. In the case of 3-HC a new vibrational signature is observed which is on the blue-side of the transition at $3268 \mathrm{~cm}^{-1}$ and is therefore potentially not a hot band. Among the possible structural isomers calculated for the monohydrated cluster ( $c f$. Fig. S3, ESI $\dagger$ ) the $\mathrm{B}^{\prime} \mathrm{T}$ and $\mathrm{A}_{\text {trans }}$ structures exhibit one very intense transition at 3349 and $3439 \mathrm{~cm}^{-1}$, respectively. The value of $3349 \mathrm{~cm}^{-1}$ of the $\mathrm{B}^{\prime} \mathrm{T}$ structure is in perfect agreement with the experimentally 
observed value, but it is questionable why the UV absorption (as observed in the R2PI spectrum after excitation of the transition at $3268 \mathrm{~cm}^{-1}$ ) of the tautomeric form does not differ from the one of the "normal" form. In the case of the $\mathrm{A}_{\text {trans }}$ structure a one-dimensional vibrational calculation performed on the potential obtained from a step-wise elongation along the $\mathrm{OH}$ stretching normal mode of the 3-HC moiety yield a value of $3354 \mathrm{~cm}^{-1}$ which is also in excellent agreement with the experimentally observed value. Thus the $\mathrm{A}_{\text {trans }}$ arrangement is an appropriate candidate to explain the additional transition at around $3340 \mathrm{~cm}^{-1}$. Although this suggestion may not be taken as a final interpretation it is a very interesting effect that the formation of a new structure is induced by an IR excitation which can be probed by a successive second IR excitation in combination with a R2PI detection. These results offer the possibilities for further developments to investigate IR induced reactions which will be analysed both experimentally and theoretically in the upcoming work.

\section{Conclusions}

In this paper 3-HC a basic unit of flavonoids is investigated as an isolated species in the gas phase. In this basic system the proton transfer reaction in the electronically excited state is not influenced by side groups. Here we present the first IR/UV analysis of 3-HC in a molecular beam for both the electronic ground and excited state. In contrast to formerly investigated systems like 3-HF and 2-NHC the analysis of 3-HC was complicated by the presence of short-lived hydrates, fragmenting down onto the monomer mass trace. This leads to an overlay of the IR vibrations of $3-\mathrm{HC}$ and hydrates in the IR/R2PI spectrum via the monomer mass trace. Since no mass signals of the clusters with water were observed under He expansion a direct comparison of the $\mathrm{S}_{0}$ state IR spectra for vibrational assignments was not possible. Therefore $\mathrm{IR}_{\text {fixed }} / \mathrm{R} 2 \mathrm{PI}$ and IR/IR/ R2PI spectroscopy were applied for the electronic ground state which did not allow concrete assignments but the conclusion that all $S_{0}$ state vibrations can be related to hydrates.

In contrast to the electronic ground state only the protontransferred monomer species could be detected in the UV/IR/ UV spectrum, which implied that the hydrates fragment after UV excitation. The presence of only the monomer species in the electronically excited state was used for further analysis: via IR/UV/IR/UV spectroscopy the band at $3461 \mathrm{~cm}^{-1}$ in the IR/R2PI spectrum could be assigned to the hydrogen-bonded $\mathrm{OH}$ stretching vibration of the 3-HC monomer, which overlaps with a hydrate vibration. A comparison with 3-HF and 2-NHC showed that the frequency of the vibration was unexpectedly high at the first sight but a simple approach with a one-dimensional potential dependent on a step-wise elongation along $\mathrm{OH}$ stretching normal mode allowed the calculation of the $\mathrm{OH}$ stretching vibration which was in good agreement with the experimentally assigned value. With respect to methodical developments it should be remarked that the quadruple IR/UV/IR/UV spectroscopy was introduced to distinguish isomers in the electronically excited state.
The application of this method to 3-HC shows that this technique can also be used to distinguish species in the electronic ground state if only vibrational transitions of one species are observed in the electronically excited state.

Thus the investigations on the basic system of the flavonols, 3-HC, show (in contrast to the substituted systems) a complicated spectral behavior which had to be analysed using a variety of sophisticated, high-level spectroscopic methods. In this context the quadruple IR/UV/IR/UV method was applied to discriminate different species in the electronic ground state. The combination of experiment and theory finally allowed a successful analysis of the 3-HC system which represents a fundamental prototype for an ESPT reaction.

\section{Acknowledgements}

The authors thank the Deutsche Forschungsgemeinschaft (DFG; Reference No. GE 961/8-1) for financial support. This work is part of the PhD thesis of M. W. and A. S.

\section{References}

1 T. P. Smith, K. A. Zaklika, K. Thakur, G. C. Walker, K. Tominaga and P. F. Barbara, J. Photochem. Photobiol., A, 1992, 65, 165.

2 G. J. Smith and K. R. Markham, J. Photochem. Photobiol., A, 1998, 118, 99.

3 S. Dennison, J. Guharay and P. K. Sengupta, Spectrochim. Acta, Part A, 1999, 55, 1127.

4 T. Schultz, Science, 2004, 306, 1765.

5 V. V. Shynkar, A. S. Klymchenko, G. Duportail, A. P. Demchenko and Y. Mély, Biochim. Biophys. Acta, Biomembr., 2005, 1712, 128.

6 Q. Chu, D. A. Medvetz and Y. Pang, Chem. Mater., 2007, 19, 6421.

7 J. Wu, W. Liu, J. Ge, H. Zhang and P. Wang, Chem. Soc. Rev., 2011, 40, 3483.

8 V. V. Shynkar, A. S. Klymchenko, E. Piémont, A. P. Demchenko and Y. Mély, J. Phys. Chem. A, 2004, 108, 8151.

9 A. P. Demchenko, K.-C. Tang and P.-T. Chou, Chem. Soc. Rev., 2013, 42, 1379.

10 J. B. Harborne and C. A. Williams, Phytochemistry, 2000, 55, 481. 11 R. Lois, Planta, 1994, 194, 498.

12 A. P. Demchenko, Trends Biotechnol., 2005, 23, 456.

13 P. F. Barbara, P. K. Walsh and L. E. Brus, J. Phys. Chem., 1989, 93, 29.

14 D. A. Parthenopoulos, D. P. McMorrow and M. Kasha, J. Phys. Chem., 1991, 95, 2668.

15 A. S. Klymchenko, T. Ozturk, V. G. Pivovarenko and A. P. Demchenko, Tetrahedron Lett., 2001, 42, 7967.

16 P. F. Barbara, P. M. Rentzepis and L. E. Brus, J. Am. Chem. Soc., 1980, 102, 2786.

17 M. Ikegami and T. Arai, J. Chem. Soc., Perkin Trans. 2, 2002, 1296.

18 P.-T. Chou, G.-R. Wu, C.-Y. Wei, C.-C. Cheng, C.-P. Chang and F.-T. Hung, J. Phys. Chem. B, 2000, 104, 7818. 
19 J. F. Ireland and P. A. H. Wyatt, Advances in Physical Organic Chemistry, 1976, p. 131.

20 P. K. Sengupta and M. Kasha, Chem. Phys. Lett., 1979, $68,382$.

21 G. J. Woolfe and P. J. Thistlethwaite, J. Am. Chem. Soc., 1981, 103, 6916.

22 B. Dick and N. P. Ernsting, J. Phys. Chem., 1987, 91, 4261.

23 G. A. Brucker and D. F. Kelley, J. Phys. Chem., 1987, 91, 2856.

24 G. A. Brucker and D. F. Kelley, J. Phys. Chem., 1988, 92, 3805.

25 B. J. Schwartz, L. A. Peteanu and C. B. Harris, J. Phys. Chem., 1992, 96, 3591.

26 A. N. Bader, V. G. Pivovarenko, A. P. Demchenko, F. Ariese and C. Gooijer, J. Phys. Chem. B, 2004, 108, 10589.

27 N. P. Ernsting and B. Dick, Chem. Phys., 1989, 136, 181.

28 A. Ito, Y. Fujiwara and M. Itoh, J. Chem. Phys., 1992, 96, 7474 .

29 K. Bartl, A. Funk and M. Gerhards, J. Chem. Phys., 2008, 129, 234306.

30 K. Bartl, A. Funk, K. Schwing, H. Fricke, G. Kock, H.-D. Martin and M. Gerhards, Phys. Chem. Chem. Phys., 2009, 11, 1173.

31 B. Dick, J. Phys. Chem., 1990, 94, 5752.

32 G. Estiúa, J. Rama, A. Pereira, R. E. Cachau and O. N. Ventura, THEOCHEM, 1999, 221.

33 J. C. Catalán, J. C. Del Valle, C. Díaz, J. Palomar, J. L. G. De Paz and M. Kasha, Int. J. Quantum Chem., 1999, 421.

34 R. Casadesús, O. Vendrell, M. Moreno, J. M. Lluch and K. Morokuma, Chem. Phys., 2006, 325, 243.

35 S. Ash, S. P. De, S. Pyne and A. Misra, J. Mol. Model., 2010, 16, 831.

36 C.-C. Hsieh, C.-M. Jiang and P.-T. Chou, Acc. Chem. Res., 2010, 43, 1364.

37 M. A. Bellucci and D. F. Coker, J. Chem. Phys., 2012, 136, 194505.

38 S. Hayaki, Y. Kimura and H. Sato, J. Phys. Chem. B, 2013, 117, 6759.

39 S. Höfener, P. C. Kooijman, J. Groen, F. Ariese and L. Visscher, Phys. Chem. Chem. Phys., 2013, 15, 12572.

40 M. Itoh, K. Tokumura, Y. Tanimoto, Y. Okada, H. Takeuchi, K. Obi and I. Tanaka, J. Am. Chem. Soc., 1982, 104, 4146.

41 D. McMorrow and M. Kasha, J. Phys. Chem., 1984, 88, 2235.

42 J. M. Petroski, C. De Sa Valente, E. P. Kelson and S. Collins, J. Phys. Chem. A, 2002, 106, 11714.

43 C. A. Kenfack, A. S. Klymchenko, G. Duportail, A. Burger and Y. Mély, Phys. Chem. Chem. Phys., 2012, 14, 8910.

44 A. Peluso, C. Adamo and G. Re, J. Math. Chem., 1992, 10, 249.

45 M. Itoh, Y. Tanimoto and K. Tokumura, J. Am. Chem. Soc., 1983, 105, 3339.

46 M. Itoh and Y. Fujiwara, J. Phys. Chem., 1983, 87, 4558.
47 M. Itoh, Y. Fujiwara, M. Sumitani and K. Yoshihara, J. Phys. Chem., 1986, 90, 5672.

48 G. A. Brucker and D. F. Kelley, J. Phys. Chem., 1987, 91, 2862. 49 M. Itoh, Pure Appl. Chem., 1993, 65, 1629.

50 K. Chevalier, M. M. N. Wolf, A. Funk, M. Andres, M. Gerhards and R. Diller, Phys. Chem. Chem. Phys., 2012, 14, 15007.

51 K. Chevalier, A. Grün, A. Stamm, Y. Schmitt, M. Gerhards and R. Diller, J. Phys. Chem. A, 2013, 117, 11233.

52 T. D. Bouman, M. A. Knobeloch and S. Bohan, J. Phys. Chem., 1985, 89, 4460.

53 H. M. Ishiki, C. Alemán and S. E. Galembeck, Chem. Phys. Lett., 1998, 287, 579.

54 S. Ash, S. P. De, H. Beg and A. Misra, Mol. Simul., 2011, $37,914$.

55 V. A. Shubert and T. S. Zwier, J. Phys. Chem. A, 2007, 111, 13283.

56 M. Weiler, K. Bartl and M. Gerhards, J. Chem. Phys., 2012, 136, 114202.

57 M. Gerhards, Opt. Commun., 2004, 241, 493.

58 M. J. Frisch, G. W. Trucks, H. B. Schlegel, G. E. Scuseria, M. A. Robb, J. R. Cheeseman, G. Scalmani, V. Barone, B. Mennucci, G. A. Petersson, H. Nakatsuji, M. Caricato, X. Li, H. P. Hratchian, A. F. Izmaylov, J. Bloino, G. Zheng, J. L. Sonnenberg, M. Hada, M. Ehara, K. Toyota, R. Fukuda, J. Hasegawa, M. Ishida, T. Nakajima, Y. Honda, O. Kitao, H. Nakai, T. Vreven, J. A. Montgomery Jr., J. E. Peralta, F. Ogliaro, M. Bearpark, J. J. Heyd, E. Brothers, K. N. Kudin, V. N. Staroverov, R. Kobayashi, J. Normand, K. Raghavachari, A. Rendell, J. C. Burant, S. S. Iyengar, J. Tomasi, M. Cossi, N. Rega, N. J. Millam, M. Klene, J. E. Knox, J. B. Cross, V. Bakken, C. Adamo, J. Jaramillo, R. Gomperts, R. E. Stratmann, O. Yazyev, A. J. Austin, R. Cammi, C. Pomelli, J. W. Ochterski, R. L. Martin, K. Morokuma, V. G. Zakrzewski, G. A. Voth, P. Salvador, J. J. Dannenberg, S. Dapprich, A. D. Daniels, Ö. Farkas, J. B. Foresman, J. V. Ortiz, J. Cioslowski and D. J. Fox, Gaussian 09, Revision D.01, Gaussian Inc., Wallingford CT, 2013.

59 Y. Nosenko, M. Kunitski, C. Riehn, R. P. Thummel, A. Kyrychenko, J. Herbich, J. Waluk and B. Brutschy, J. Phys. Chem. A, 2008, 112, 1150.

60 Y. Nosenko, M. Kunitski, C. Riehn, P. H. P. Harbach, A. Dreuw and B. Brutschy, Phys. Chem. Chem. Phys., 2010, 12, 863 .

61 A. Douhal, F. Lahmani, A. Zehnacker-Rentien and F. Amat-Guerri, J. Phys. Chem., 1994, 98, 12198.

62 A. Funk, T. Martin and M. Gerhards, in preparation for publication.

63 B. C. Dian, A. Longarte, P. R. Winter and T. S. Zwier, J. Chem. Phys., 2004, 120, 133. 